\title{
Study on viscosity modification of human and formula milk for infants with dysphagia
}

\author{
Mariangela Bartha de Mattos Almeida ${ }^{(1)}$ \\ Saint Clair Gomes Júnior ${ }^{(1)}$ \\ Jonas Borges da Silva ${ }^{(1)}$ \\ Danielle Aparecida da Silva ${ }^{(1)}$ \\ Maria Elisabeth Lopes Moreira ${ }^{(1)}$
}

(1) Instituto Nacional de Saúde da Mulher, da Criança e do Adolescente Fernandes Figueira - IFF/ Fundação Oswaldo Cruz FIOCRUZ - Rio de Janeiro (RJ) - Brazil. Research support source: FAPERJ

Conflict of interest: Nonexistent

Received on: April 27, 2017

Accepted on: September 5, 2017

Mailing address:

Mariangela Bartha de Mattos Almeida Instituto Nacional de Saúde da Mulher,

da Criança e do Adolescente Fernandes

Figueira - IFF/Fiocruz

Setor de Fonoaudiologia Hospitalar

Av. Rui Barbosa, 716 - Flamengo -

Rio de Janeiro, Rio de Janeiro, Brasil CEP: 22250-020

E-mail: marianbartha@iff.fiocruz.br

\section{ABSTRACT}

Purpose: to analyze the modification of the viscosity of human milk and infant formula. Methods: three studies were performed to assess the viscosity and effect of time on infant formula with a thickener, at concentrations of 2, 3, and $5 \%$, as well as raw and pasteurized human milk at concentrations of $2,3,5$, and $7 \%$ at $37^{\circ} \mathrm{C}$, for 60 minutes. Rice cereal was used as a thickening agent. The viscosity was evaluated using a Ford Cup-type viscometer, and the samples were analyzed at 20-minute intervals. Significant differences were assessed using the ANOVA test.

Results: no significant differences in viscosity were observed over time in concentrations of 2,3 , and $5 \%$. There was a difference in the viscosity between human milk and infant formula, in concentrations of $2 \%$ and $5 \%, 2 \%$ and $7 \%, 3 \%$ and $5 \%$, and $3 \%$ and $7 \%$, independently of the time intervals evaluated.

Conclusion: the findings of this study demonstrate the need for different concentrations of the thickening agent for human milk and infant formula. Rice cereal is a suitable therapeutic option for newborns presented with dysphagia in concentrations of 2 , 3,5 , and $7 \%$, due to its effect on the viscosity and flow reduction, provided that the feeding time is considered.

Keywords: Human Milk; Thickeners; Dysphagia; Deglutition Disorders; Viscosity 


\section{INTRODUCTION}

Dysphagia is defined as difficulty in eating (from Greek $d y s=$ difficulty, and phagos = eating) and can result in a diversion in the food path, thereby partially or totally blocking the airways. It is a symptom of a baseline disease that is most often transient, affecting full-term and preterm newborn infants who present neurological, respiratory, cardiac, and gastric diseases; the disease can also be caused by immaturity. The causes may be neurogenic or mechanical and the consequences may compromise the preparatory, oral, pharyngeal, or esophageal phases, thus posing serious risks to the health of the newborn ${ }^{1-3}$.

Studies suggest that the prevalence of dysphagia is up to $60 \%$ in the NICU population, whereas the incidence is in the range of 25 to $45 \%$ in children developing normally and 30 to $80 \%$ in children with developmental disorders. About 37 to $40 \%$ of children with eating difficulties had been born prematurely and present a risk of incoordination of swallowing due to the immaturity of their systems and the presence of comorbidities, such as bronchodysplasia, chronic pulmonary diseases, congenital abnormalities, and gastroesophageal reflux disease ${ }^{5-7}$. However, these data are limited and show great variability due to factors related to the selection of the participants, the definition of the disorder, and the choice of evaluation methods.

Modifications in the viscosity of liquids by means of thickeners have been used as therapy to improve swallowing in newborns. The increase in milk viscosity causes a change in its consistency, with a consequent alteration in the flow. This makes early escape to the oropharynx difficult and allows better management of the alimentary bolus and coordination of suction, breathing, and swallowing ${ }^{8-10}$.

Control of the viscosity of modified diets is clinically important to minimize the risk of aspiration in the management and treatment of dysphagia ${ }^{10,11}$. Viscosity is a property that characterizes a fluid and allows measurement of its internal resistance to its displacement at the surface ${ }^{10}$. For newborns, the diet has a thin consistency, which requires greater control of the proprioception, organization, and coordination between suction, breathing and swallowing. Diet with modified viscosity favors coordination between the processes of suction, breathing, and swallowing, and, therefore, the baby is better prepared for swallowing ${ }^{12}$.

A study by Almeida et al. ${ }^{13}$ showed different results for viscosity depending on the type of milk used. The thickening agent used was cornstarch with maltodextrin.
When added to human milk, this thickener induced a decrease in viscosity over time, whereas it facilitated an increase in viscosity when added to infant milk formula. Therefore, we concluded that viscosity is related to time, and demonstrates different patterns depending on the type of milk and the thickening agent used ${ }^{14-16}$.

Therefore, this study aims to analyze the viscosity of human milk and infant milk formula thickened with rice cereal at different concentrations over a period of 60 minutes at $37 \circ-\mathrm{C}$.

\section{METHODS}

An experimental study was conducted to analyze the effect of time on the viscosity of human milk and milk formula thickened at different concentrations of the thickening agent in a 60 -minute period at $37^{\circ} \mathrm{C}$. The study was conducted in the Quality Control Laboratory of the Human Milk Bank (HMB), and approval was obtained from the Committee of Ethics of Research with Human Beings of the IFF/Fiocruz under no. 543,616. All samples of human milk under study came from donors registered in the HMB-IFF, observing the provisions in the legislation that regulate the implantation and operation of human milk banks ${ }^{17}$.

Guidelines of good handling practices were followed for all milk samples donated to and collected by the Human Milk Bank (HMB). In the HMB, human milk undergoes a selection and classification process, where it is separated into colostrum, transition milk, and mature milk, as well as by acidity and calorie content. The acidity and calorie content of the human milk samples selected for this study were between 1 and 4 D and between 500 and $650 \mathrm{kcal} / \mathrm{l}$, respectively.

Three experiments were performed to assess the viscosity and the effect of time after the addition of a thickener. The first involved analysis of the milk formula; the second, evaluation of the raw pumped human milk (RPHM); and the third, evaluation of the pasteurized pumped human milk (PPHM).

The modified milk formula was analyzed at thickener concentrations of 2,3 , and $5 \%$, while pumped human milk (raw or pasteurized) was analyzed at thickener concentrations of $2,3,5$, and $7 \%$. All experiments were performed in triplicate using $150 \mathrm{ml}$ and $200 \mathrm{ml}$ of each formula and pumped human milk sample, respectively. A total of 33 samples were analyzed, which were subdivided into 9 samples for the formula and 24 samples for the pumped human milk analyses. For the analyzed samples, 36 measurements were performed for 
experiments with the formula and 96 measurements, for the human milk.

The thin consistency used in this study is based on the standard indicated by the speech-language pathology department of the institution. It is justified by its ability to enable better suction in the bottle or cup and because it represents a considerable increase in the viscosity of milk for the newborn population with dysphagia.

The concentrations used are part of the routine process followed by the department and vary according to the degree of severity of dysphagia ${ }^{18}$ diagnosed by the speech-language pathology team. The thickening agent used was the Vitalon ${ }^{\circledR}$ rice cereal (WOW Nutrition, São Paulo, Brazil), which has the advantage of lacking traces of milk in its composition, and which is therefore recommended by the speech-language pathology team of the institution.

Each sample was analyzed four times at 20-minute intervals within an hour. In the experiment using human milk, each test was performed separately: the defrosting of the milk was carried out first, followed by pouring by means of a beaker. The quality of the pasteurized samples complied with the recommendations of the National Health Surveillance Agency (ANVISA) ${ }^{19}$.

The samples were kept in a water bath at $37^{\circ} \mathrm{C} \pm$ $2^{\circ} \mathrm{C}$. A calibrated glass liquid thermometer was used to verify the thermal stability to allow for the addition of the thickener. All samples were shaken during preparation of the thickened milk for better solubilization, as recommended by the manufacturer.

The viscosity was evaluated with the Ford Cup-type viscometer Quimis brand model Q 280, which analyzes the kinematic viscosity expressed in centiStoke (cSt) by using orifice 2 for all concentrations, according to Brazilian standard NBR $5849^{20}$. For all evaluations, the formula $\left[(2,388 \times t)-\left(0,007 \times t^{2}\right)-57,008\right]$ was applied, where $t$ represents the flow time of the fluid in seconds. The concentration of $7 \%$ was not analyzed for the formula because its viscosity made it impossible to pass through orifice 2 . The process of determining the viscosity of human milk using the Ford Cup has been previously described ${ }^{13}$.

The viscosity table recommended by the American Dietetic Association ${ }^{18}$ was used to adjust the viscosity parameters to a thin consistency (1-50 cP), which is adequate for the study population and allows suction through a bottle or cup feeding.

Descriptive analyses were performed using the means and standard deviation of the variables assessed. The distribution normality was verified by Kolmogorov-Smirnov (KS) and Shapiro-Wilk tests. Analysis of variance (ANOVA) was used to verify significant differences between the different degrees of thickening. A post hoc test, with Tukey distribution, was performed to identify pairs with statistically significant differences. All analyses were performed using the SPSS 21 software, with a significance level of 0.05 .

\section{RESULTS}

In the analysis of viscosity behavior over time with the milk formula (Table 1), there were no significant differences in viscosity between the time intervals at the different concentration levels evaluated ( $p$-value 0.277 , 0.263 , and 0.065 , respectively). There was a significant difference in the viscosity values between concentrations of $3 \%$ and $5 \%(p<0.0001)$ and $2 \%$ and $5 \%(p$ $<0.0001$ ), regardless of the time period analyzed.

Table 1. Effects of time on the viscosity of standard milk formula, thickened and incubated at $37^{\circ} \mathrm{C}$

\begin{tabular}{cccc}
\hline Time & $\mathbf{V}$ (cSt) & $\mathbf{V}$ (cSt) & $\mathbf{V}$ (cSt) \\
(minutes) & $\mathbf{2 \%}$ & $\mathbf{3 \%}$ & $\mathbf{5 \%}$ \\
\hline 0 & $4.17 \pm 0.99$ & $7.37 \pm 0.81$ & $18.31 \pm 3.69$ \\
20 & $5.13 \pm 1.19$ & $7.73 \pm 1.24$ & $23.82 \pm 3.87$ \\
40 & $5.26 \pm 1.21$ & $8.15 \pm 0.57$ & $25.98 \pm 2.62$ \\
60 & $5.97 \pm 0.63$ & $8.73 \pm 0.20$ & $25.80 \pm 2.69$ \\
\hline$p$-value & 0.277 & 0.263 & 0.065 \\
\hline
\end{tabular}

$\mathrm{V}=\mathrm{Viscosity} ; \mathrm{cSt}=$ CentiStoke (unit of kinematic viscosity)

p-value $2 \%$ vs $3 \%-0.072$

p-value $2 \%$ vs $5 \%-0.000$

p-value $3 \%$ vs $5 \%-0.000$ 
In the experiment with raw human milk (Table 2), no significant differences were observed for viscosity over time at thickener concentrations of 2,3 , and $5 \%$ (p-value of $0.630,0.850$, and 0.825 , respectively). At the concentration of $7 \%$, a variation in viscosity was observed with respect to time ( $p$-value of 0.031 ). Significant differences in viscosity values were found between concentrations of $2 \%$ and $5 \%$ (p-value $<0.0001$ ), $2 \%$ and $7 \%$ (p-value $<0.0001$ ), $3 \%$ and $5 \%$ (p-value $<0.0001$ ), and $3 \%$ and $7 \%(p<0.0001)$, independent of the time interval analyzed.

Table 2. Effect of time on the viscosity of raw human milk thickened and incubated at $37^{\circ} \mathrm{C}$

\begin{tabular}{ccccc}
\hline Time & $\mathbf{V}$ (cSt) & $\mathbf{V}$ (cSt) & $\mathbf{V}$ (cSt) & $\mathbf{V}$ (cSt) \\
(minutes) & $\mathbf{2 \%}$ & $\mathbf{3 \%}$ & $\mathbf{5 \%}$ & $\mathbf{7 \% *}$ \\
\hline 0 & $4.44 \pm 0.62$ & $4.38 \pm 1.74$ & $7.29 \pm 2.35$ & $10.14 \pm 0.52$ \\
20 & $4.46 \pm 1.61$ & $4.20 \pm 1.07$ & $7.06 \pm 1.44$ & $9.33 \pm 0.57$ \\
40 & $3.60 \pm 0.30$ & $3.61 \pm 0.60$ & $7.26 \pm 0.93$ & $8.84 \pm 0.51$ \\
60 & $4.02 \pm 0.48$ & $4.15 \pm 0.70$ & $6.26 \pm 0.95$ & $8.88 \pm 0.17$ \\
\hline p-value & 0.630 & 0.850 & 0.825 & 0.031 \\
\hline
\end{tabular}

$\mathrm{V}=$ Viscosity; $\mathrm{cSt}=$ CentiStoke (unit of kinematic viscosity)

p- value $2 \%$ vs $3 \%-0.899 ; p$ - value $3 \%$ vs $5 \%-0.000$

p- value $2 \%$ vs $5 \%-0.000 ; p$ - value $3 \%$ vs $7 \%-0.000$

$p$ - value $2 \%$ vs $7 \%-0.000 ; p$ - value $5 \%$ vs $7 \%-0.000$

*differences only observed between 0 and 40 minutes ( $p$-value of 0.016 ) and 0 and 60 minutes ( $p$-value of 0.036 )

In the experiment with pasteurized human milk (Table 3), no significant differences in viscosity were identified over time at thickener concentrations of 2, 3, and $5 \%$ ( $p$-value $0.721,0.136$, and 0.709 , respectively). A change in viscosity over time for the concentration of $7 \%$ ( $p$-value of 0.028 ) was observed. Significant differences were observed in the viscosity values between concentrations of $2 \%$ and $5 \%(p<0.0001), 2 \%$ and $7 \%(p<0.0001), 3 \%$ and $5 \%(p<0.0001)$, and $3 \%$ and $7 \%(p<0.0001)$, regardless of the time interval at which the analysis was conducted.

Table 3. Effect of time on the viscosity of pasteurized human milk thickened and incubated at $37^{\circ} \mathrm{C}$

\begin{tabular}{ccccc}
\hline Time & $\mathbf{V}$ (cSt) & $\mathbf{V}$ (cSt) & $\mathbf{V}$ (cSt) & $\mathbf{V}$ (cSt) \\
(minutes) & $\mathbf{2 \%}$ & $\mathbf{3 \%}$ & $\mathbf{5 \%}$ & $\mathbf{7 \%}^{*}$ \\
\hline 0 & $4.73 \pm 1.37$ & $3.83 \pm 0.71$ & $8.21 \pm 1.05$ & $11.73 \pm 1.49$ \\
20 & $4.11 \pm 0.49$ & $4.70 \pm 0.57$ & $7.62 \pm 0.99$ & $9.26 \pm 0.90$ \\
40 & $4.73 \pm 0.51$ & $4.76 \pm 0.17$ & $7.34 \pm 1.53$ & $8.84 \pm 0.87$ \\
60 & $4.88 \pm 0.85$ & $4.79 \pm 0.42$ & $7.04 \pm 1.37$ & $9.08 \pm 0.66$ \\
\hline p-value & 0.721 & 0.136 & 0.709 & 0.028 \\
\hline
\end{tabular}

$\mathrm{V}=$ Viscosity; $\mathrm{cSt}=$ CentiStoke (unit of kinematic viscosity)

p-value $2 \%$ vs $3 \%-0.757$; p-value $3 \%$ vs $5 \%-0.000$

$p$-value $2 \%$ vs $5 \%-0.000 ; p$-value $3 \%$ vs $7 \%-0.000$

$p$-value $2 \%$ vs $7 \%-0.000 ; p$-value $5 \%$ vs $7 \%-0.000$

* differences only observed between 0 and 40 minutes ( $p$-value of 0.044 ) and 0 and 60 minutes ( $p$-value of 0.048 ) 
When comparing the mean viscosity values of raw and pasteurized human milk with $5 \%$ thickener concentration with the milk formula with $3 \%$ thickener concentration (7.29 cSt, $8.21 \mathrm{cSt}$ and $7.37 \mathrm{cSt}$, respectively), no significant differences were observed between raw human milk and the formula ( $p$-value $=0.228$ ) and between pasteurized human milk and formula ( $p$-value $=0.561$ ), regardless of the time interval at which the analysis was conducted.

In each of the three experiments, no significant differences in viscosity values between the concentrations of $2 \%$ and $3 \%(p>0.05)$ were observed, regardless of time.

\section{DISCUSSION}

Control of the viscosity of milk is an important and challenging task for speech-language pathologists who deal with dysphagia. It should be carried out only when all other stimulation approaches have not had the desired effect, that is, to promote safe oral feeding. In addition, such a strategy should be used transiently, while the newborn acquires oral skills to feed in a coordinated way.

The main finding of this study was the validation that human milk and formula, thickened with rice cereal, can maintain their stability for 1 hour, a period considered as the expected limit to end the feeding of a newborn with dysphagia. These newborns are normally fed a formula with thickeners or commercial additives based on gum, algae, cellulose, and other components that are difficult to digest and can cause adverse effects. There is therefore the option of using human milk in such cases ${ }^{11}$.

In view of the recommendations from new studies ${ }^{12}$, and the results reported by Almeida et al. ${ }^{13}$, who demonstrated a significant change in viscosity and time with maize starch and maltodextrin as thickening agents, we decided to evaluate whether rice cereal would be more stable when added to human milk and milk formula. The results showed that the viscosity of human milk and formula remained stable for 1 hour since the use of a rice cereal-thickening agent. However, at a thickener concentration of $7 \%$ at 20 minutes of flow of the pumped human milk, there was a decrease in the viscosity. This means that health professionals involved with the feeding of newborns with dysphagia should be especially aware of the feeding time with milks thickened at higher concentrations.

Using rice cereal as a thickener is advantageous because it is based on instant soluble starch, which is easy to prepare, and does not contain traces of milk in its composition. It is also inexpensive and is found easily in the market. Temporal stability, with the viscosity remaining the same over time, has the advantage of allowing newborns that need more time to develop their diet to easily extract the milk until the end of the feed.

Significant differences were observed between human milk and formula at the concentrations evaluated. An increase in the viscosity of milk formula samples was observed from $3 \%$ onwards, and higher viscosity values were observed compared to human milk with the same thickener concentration. This can be explained by the presence of amylase enzyme in human milk, which, even after pasteurization, can cause partial hydrolysis of the starch and consequently, a decrease in viscosity ${ }^{13}$. This effect positively correlated with increased concentration.

Similar viscosity was observed between milk formula with $3 \%$ thickener and human milk with $5 \%$ thickener. This allows flexibility of feeding to the mother after discharge from the hospital, in case it is necessary to change the milk to feed her baby. In this case, the team should provide guidance to the mother with regards to the necessary adjustments.

Although the Ford Cup-type viscometer is a traditional instrument that is easy to handle and for control of the kinematic viscosity of fluids, it can be considered one of the limitations of this study. This is because it is a dependent operator, which could introduce an important measurement bias. To minimize this bias, we used three milk samples for each of the concentrations considered. Therefore, it is believed that measurement errors have been minimized in this study.

Although the choice of temperature of milk is related to body temperature, it is a parameter that is difficult to maintain on a daily basis in a Neonatal ICU environment, requiring that the diet be consumed by the newborn immediately after its preparation, to minimize its effect.

Further studies are needed to analyze the osmolarity and bioavailability of the nutrients of milk and of a formula after the thickening. Developing guidelines for thickening agents that allow the use of human milk by newborns at risk for dysphagia is also recommended, in order to facilitate oral feeding as well as avoid alternative feeding and malnutrition. 


\section{CONCLUSION}

This study demonstrated that the modified viscosity from addition of the rice cereal depends on the concentration and the type of milk used. The flow time had a significant influence on human milk at a $7 \%$ concentration, after 20 minutes. The rice cereal thickener is a therapeutic option at concentrations of 2, 3, 5, and $7 \%$ for newborns with dysphagia provided that the appropriate time of feeding is observed.

\section{ACKNOWLEDGMENTS}

We would like to thank the mothers who made this study possible by donating their human milk.

\section{REFERENCES}

1. Levy DS, Rainho L. Abordagem em disfagia infantil - Proposta fonoaudiológica e fisioterápica. In: Jacobi JS, Levy DS, Silva LMC. Disfagia: avaliação e tratamento. Editora Revinter; Rio de Janeiro: 2003. p. 37-65.

2. Dusick A. Investigation and management of dysphagia. Seminars in Pediatric Neurology. 2003;10(4):255-64.

3. Arvedson JC. Evaluation of children with feeding and swallowing problems. Language, Speech and Hearing Services in the Schools. 2000;31(1):28-41.

4. Jadcherla SR, Shubert TR, Gulati IK, Jensen PS, Wei L, Shaker R. Upper and lower esophageal sphincter kinetics are modified during maturation: effect of pharyngeal stimulus in premature infants. Pediatric Research. 2015;77(1) 99-106.

5. Arvedson JC. Assessment of pediatric dysphagia and feeding disorders: Clinical and instrumental approaches. Developmental Disabilities Research Reviews. 2008;14(2):118-27.

6. Lefton-Greif MA. Pediatric dysphagia. Physical medicine and rehabilitation clinics of North America. 2008;19(4):837-51.

7. Brackett K, Arvedson JC, Manno CJ. Pediatric feeding and swallowing disorders: General assessment and intervention. Perspectives on Swallowing and Swallowing Disorders. 2006;15(3):10-4.

8. Matta Z, Chambers E, Garcia JM, Helverson JM. Sensory characteristics of beverages prepared with commercial thickeners used for dysphagia diets. J Am Diet Assoc. 2006;106(7):1049-54.

9. Adeleye B, Rachal C. Comparison of the rheological properties of ready-to-serve and powdered instant food-thickened beverages at different temperaturas for dysphagic patients. J Am Diet Assoc. 2007;107(7):1176-82

10. Groher ME. Dysphagia: diagnosis and management. Copyrighted Material; 1997.

11. Souza BB, Martins C, Campos DJ, Balsini ID, Meyer LR. Nutrição e disfagia: guia para profissionais. Nutroclínica; Curitiba: 2003.

12. Gosa M, Schooling T, Coleman J. Thickened liquids as a treatment for children with dysphagia and associated adverse effects - A systematic review. Infant, Child, \& Adolescent Nutrition. 2011;3(6):344-50.

13. Almeida MBM, Almeida JAG, Moreira MEL, Novak FR. Adequacy human milk viscosity to respond to infants with dysphagia: Experimental Study. Journal of Applied Oral Science. 2011;19(6):554-9.

14. Garcia JM, Chambers E. Managing dysphagia through diet modifications. American Journal of Nursing. 2010;110(11):26-33.

15. Lotong V, Chun SS, Chambers E, Metz JG. Texture and flavor characteristics of beverages containing commercial thickening agents for dysphagia diets. Journal of Food Science. 2003;68(4):1537-41.

16. Madhoun LL, Siler-Wurst KK, Sitaram S, Jadcherla SR. Feed-thickening practices in NICUs in the current era: Variability in prescription and implementation patterns. Journal of Neonatal Nursing. 2015;21(6):255-62.

17. Agência Nacional de Vigilância Sanitária (ANVISA). Resolução RDC no171 dispõe sobre o regulamento, a implantação e o funcionamento de Bancos de Leite Humano no território nacional. Diário Oficial da União de 04/09/2006. Brasil.

18. American Dietetic Association (ADA). The national dysphagia diet: standardization for optimal care. Chicago, USA; 2002.

19. Agência Nacional de Vigilância Sanitária (ANVISA). Banco de leite humano: funcionamento, prevenção e controle de riscos. Brasília: 2008.

20. Associação Brasileira de Normas Técnicas (ABNT). Norma NBR 5849: Determinação da viscosidade pelo Copo Ford. Rio de Janeiro; 2ª edição, 2015. 